\title{
FACTORIZATION IN OPERATOR ALGEBRAS
}

\author{
BARUCH SOLEL
}

(Communicated by John B. Conway)

\begin{abstract}
Let $M \subseteq B(H)$ be a $\sigma$-finite von Neumann algebra and let $\mathcal{L}$ be a lattice of projections associated with a unitary representation $\left\{W_{t}\right\}$ of a compact group with an ordered dual. ( $\mathcal{L}$ is not necessarily contained in $M$.) Assume that $M$ is invariant under ad $W_{t}$ for every $t$.

Then, whenever $T$ is an invertible operator in $M$ that can be factored as $T=U A$ where $U$ is a unitary operator in $B(H)$ and $A$ lies in alg $\mathcal{L} \cap(\operatorname{alg} \mathcal{L})^{-1}$, then $U$ and $A$ can be chosen in $M$.

As corollaries we derive results about factorization with respect to CSL subalgebras and analytic subalgebras of von Neumann algebras.
\end{abstract}

1. Introduction. Let $M$ be a $\sigma$-finite von Neumann algebra and $\tau \subseteq M$ be a $\sigma$-weakly closed (nonselfadjoint) subalgebra of $M$. The factorization problem with respect to $(M, \tau)$ is the following problem.

Given an invertible operator $T$ in $M$, can we write $T=U A$ where $U$ is a unitary operator in $M$ and $A$ lies in $\tau \cap \tau^{-1}$ ?

This problem is closely related to the problem of similarity among algebras of operators (see [5 and 2]). Consider the following example. Let $\mathbf{T}$ denote the unit circle, $H$ be $L^{2}(\mathrm{~T}),\{e\}_{n=-\infty}^{\infty}$ be the usual orthonormal basis $e_{n}=z^{n}$ and $p_{j}$ be the projection onto the closed subspace spanned by $\left\{e_{k}: k \geq j\right\},-\infty<j<-\infty$. Write $\mathcal{L}$ for the nest $\left\{p_{j}: j \in \mathbf{Z}\right\}$ and alg $\mathcal{L}$ for the associated nest algebra $\{T \in$ $B(H):\left(1-p_{j}\right) T p_{j}=0$ for all $\left.j\right\}$. It follows from Arveson's result [2, Theorem $3.3]$ that, in this case, every invertible operator $T$ in $B(H)$ can be factored with respect to $(B(H)$, alg $\mathcal{L})$. Let $M \subseteq B\left(L^{2}(\mathbf{T})\right)$ be the algebra of all the operators of multiplication by $L^{\infty}$ functions and let $T$ be an invertible operator in $M$. Since $M \cap \operatorname{alg} \mathcal{L}$ is the algebra of all the operators of multiplication by $H^{\infty}$ functions, we know from the classical theory of $H^{\infty}(\mathbf{T})$ that, in fact, $T$ can be factored with respect to $(M, M \cap \operatorname{alg} \mathcal{L})$.

It is natural to ask whether this situation is more general. To state it more precisely, let $\mathcal{L}$ be a lattice of projections in $B(H)$, let $T$ be an invertible operator that can be factored with respect to $(B(H)$, alg $\mathcal{L}$ ) (where alg $\mathcal{L}=\{T \in B(H)$ : $(1-P) T P=0$ for all $P$ in $\mathcal{L}\})$ and let $M$ be a von Neumann algebra that contains $T$. Can $T$ be factored with respect to $(M, M \cap \operatorname{alg} \mathcal{L})$ ?

Received by the editors December 1, 1986.

1980 Mathematics Subject Classification (1985 Revision). Primary 46L10, 47C15; Secondary $47 \mathrm{D} 25$.

Key words and phrases. Invertible operator, factorization, analytic subalgebra, CSL subalgebra, von Neumann algebra. 
In this paper we prove that if $\mathcal{L}$ is a subspace lattice associated with a unitary representation $\left\{W_{t}: t \in A\right\}$ of a compact abelian group $A$ with an ordered dual group $\Gamma$ and if $M$ is invariant under ad $W_{t}$ (i.e. $W_{t} M W_{t}^{*}=M$ ) for all $t \in A$, then whenever $T$ can be factored with respect to $(B(H)$, alg $\mathcal{L})$, it can be factored with respect to $(M, M \cap \operatorname{alg} \mathcal{L})$.

As corollaries we derive results about factorization with respect to CSL subalgebras and analytic operator algebras.

For more about the factorization problem and other related problems see $[\mathbf{1}, \mathbf{2}$, $3,5,6,8,9,11]$.

We will assume that all Hilbert spaces are complex and separable and $B(H)$ will denote the algebra of all bounded operators on the Hilbert space $H$. If $S \subseteq H$ is a set of vectors, $[S]$ will denote the closed subspace spanned by $S$.

2. The factorization results. Let $A$ be a compact abelian group with dual $\Gamma$. Let $W=\left\{W_{t}: t \in A\right\}$ be a strongly continuous unitary representation of $A$ on a Hilbert space $H$. By Stone's Theorem, there is a unique projection valued measure $P(\cdot)$ on the Borel sets of $\Gamma$ such that

$$
W_{t}=\int_{\Gamma}\langle t, p\rangle d P(p), \quad t \in A,
$$

where $\langle t, p\rangle$ is the dual pairing of $A$ and $\Gamma$. Let $\Sigma \subseteq \Gamma$ be a positive semigroup; i.e. $\Sigma+\Sigma \subseteq \Sigma$ and $\Sigma \cap(-\Sigma)=\{0\}$.

A lattice of projections in $B(H)$ is called a subspace lattice if it contains 0 and $I$ and is closed in the strong operator topology. Write $\mathcal{L}$ for the (commutative) subspace lattice generated by $\{P(q+\Sigma): q \in \Gamma\}$. Also let $\alpha_{t}, t \in A$, be the automorphism implemented by $W_{t}$, i.e. $\alpha_{t}(T)=W_{t} T W_{t}^{*}, T \in B(H)$.

THEOREM. Suppose $T=U A$ where $U$ is a unitary operator in $B(H)$ and $A$ lies in $\operatorname{alg} \mathcal{L} \cap(\operatorname{alg} \mathcal{L})^{-1}$.

Then $T=W B$ where $W$ is a unitary operator, $B$ lies in $(\operatorname{alg} \mathcal{L}) \cap(\operatorname{alg} \mathcal{L})^{-1}$ and both $W$ and $B$ lie in the smallest $\left\{\alpha_{t}\right\}$-invariant von Neumann algebra that contains $T$.

Proof. We have $T=U A, A \in \operatorname{alg} \mathcal{L} \cap(\operatorname{alg} \mathcal{L})^{-1}$ and $U$ is a unitary operator in $B(H)$. Write $M$ for the smallest $\left\{\alpha_{t}\right\}$-invariant von Neumann algebra that contains $T$. For $q \in \Gamma,[A P(q+\Sigma) H]=[P(q+\Sigma) H]$; hence $[T P(q+\Sigma) H]=U[P(q+\Sigma) H]$. Since $\alpha_{t}(M)=M, t \in A$, we have $\alpha_{t}\left(M^{\prime}\right)=M^{\prime}$; so that $\alpha$ defines a compact action on $M^{\prime}$. Write $R_{q}=\left\{S \in M^{\prime}: \alpha_{t}(S)=\langle t, q\rangle S\right.$ for every $\left.t \in A\right\}, q \in \Gamma$. It is known that the $\sigma$-weakly closed span of $\bigcup_{q \in \Gamma} R_{q}$ is $M^{\prime}$. Also, if $S \in R_{q}$ and $S=V|S|$ is its polar decomposition then $V \in R_{q}$ and $|S| \in R_{0}$. Now fix $q \in \Gamma$ and a partial isometry $V$ in $R_{q}$. Then

$$
W_{t} V W_{t}^{*}=\langle t, q\rangle V, \quad t \in A .
$$

Then $V^{*} W_{t} V=\langle t, q\rangle V^{*} V W_{t}$; hence

$$
\int_{\Gamma}\langle t, p\rangle d\left(V^{*} P(\cdot) V\right)(p)=\int_{\Gamma}\langle t, p+q\rangle V^{*} V d P(p)=\int_{\Gamma}\langle t, p\rangle V^{*} V d P(p-q) .
$$


Therefore,

$$
V^{*} P(\gamma+\Sigma) V=V^{*} V P(\gamma-q+\Sigma), \quad \gamma \in \Gamma .
$$

Let $Q(\cdot)$ be the projection valued measure defined by $Q(\cdot)=U P(\cdot) U^{*}$ and write $U_{t}=\int_{\Gamma}\langle t, p\rangle d Q(p)=U W_{t} U^{*}, t \in A$. We have

$$
\begin{aligned}
V^{*}[Q(\gamma+\Sigma) H] & =V^{*}[U P(\gamma+\Sigma) H]=V^{*}[T P(\gamma+\Sigma) H] \\
& =\left[T V^{*} P(\gamma+\Sigma) H\right]=\left[T V^{*} V P(\gamma-q+\Sigma) H\right] \\
& =V^{*} V[Q(\gamma-q+\Sigma) H] .
\end{aligned}
$$

Hence $V^{*} Q(\gamma+\Sigma) V=V^{*} V Q(\gamma-q+\Sigma), \gamma \in \Gamma$.

Integrating, we get for $t \in A$,

$$
\begin{aligned}
V^{*} U_{t} V & =\int\langle t, p\rangle d\left(V^{*} Q(p) V\right)(p)=\int\langle t, p\rangle V^{*} V d Q(p-q) \\
& =\int\langle t, p\rangle\langle t, q\rangle V^{*} V d Q(p)=\langle t, q\rangle V^{*} V U_{t} .
\end{aligned}
$$

For $q=0$ and $V$ a unitary operator in $R_{0}\left(=\left\{S \in M^{\prime}: \alpha_{t}(S)=S, t \in A\right\}\right)$ we get $V^{*} U_{t} V=U_{t}$; hence $U_{t} \in R_{0}^{\prime}$. For a partial isometry $V$ in $R_{q}, q \in \Gamma$, we now have

$$
U_{t} V U_{t}^{*}=U_{t} V V^{*} V U_{t}^{*}=V V^{*} U_{t} V U_{t}^{*}=\langle t, q\rangle V V^{*} V=\langle t, q\rangle V=\alpha_{t}(V) .
$$

Hence, for every $S \in M^{\prime}, U_{t} S U_{t}^{*}=\alpha_{t}(S)=W_{t} S W_{t}^{*}$.

Now write

$$
\tilde{W}_{t}=\left(\begin{array}{cc}
W_{t} & 0 \\
0 & U_{t}
\end{array}\right) \in B(H \oplus H)
$$

and

$$
\tilde{R}=\left\{\left(\begin{array}{cc}
S & 0 \\
0 & S
\end{array}\right): S \in M^{\prime}\right\} \subseteq B(H \oplus H) .
$$

From the above it follows that $\tilde{W}_{t} \tilde{R} \tilde{W}_{t}^{*}=\tilde{R}$. Hence also $\tilde{W}_{t}(\tilde{R})^{\prime} \tilde{W}_{t}^{*}=\tilde{R}^{\prime}$. But

$$
\tilde{R}^{\prime}=\left\{\left(\begin{array}{ll}
S_{11} & S_{12} \\
S_{21} & S_{22}
\end{array}\right): S_{i j} \in M\right\} .
$$

Let $\gamma_{t}$ be ad $\tilde{W}_{t}$; i.e., $\gamma_{t}(S)=\tilde{W}_{t} S \tilde{W}_{t}^{*}$ for $S \in B(H \oplus H)$ and write $\varepsilon(S)=$ $\int_{A} \gamma_{t}(S) d t$. Then $\varepsilon$ is a faithful normal expectation from $B(H \oplus H)$ onto $\{S \in$ $\left.B(H \oplus H): \gamma_{t}(S)=S, t \in A\right\}$ and $\varepsilon\left(\tilde{R}^{\prime}\right)=\left\{S \in \tilde{R}^{\prime}: \gamma_{t}(S)=S, t \in A\right\}$. We have $\tilde{W}_{t}=\int_{\Gamma}\langle t, p\rangle d \tilde{P}(p)$ where

$$
\tilde{P}(E)=\left(\begin{array}{cc}
P(E) & 0 \\
0 & Q(E)
\end{array}\right)
$$

and if we write $\tilde{\mathcal{L}}$ for the lattice

$$
\left\{\left(\begin{array}{cc}
P(q+\Sigma) & 0 \\
0 & Q(q+\Sigma)
\end{array}\right): q \in \Gamma\right\}
$$

then alg $\tilde{\mathcal{L}}=\left\{S \in B(H \oplus H): \operatorname{sp}_{\gamma}(S) \subseteq \Sigma\right\}$ where $\operatorname{sp}_{\gamma}(\cdot)$ is Arveson's spectrum (see [4, Proposition 2.1]).

Also note that $\varepsilon$, restricted to alg $\tilde{\mathcal{L}}$, is multiplicative. 
Now write

$$
\tilde{T}=\left(\begin{array}{cc}
0 & T^{-1} \\
T & 0
\end{array}\right) \in \tilde{R}^{\prime}
$$

Since $\left(\begin{array}{ll}I & 0 \\ 0 & 0\end{array}\right)$ is in the range of $\varepsilon$ and $\varepsilon$ is an expectation we get $\varepsilon(\tilde{T})=\left(\begin{array}{cc}0 & T_{1} \\ T_{0} & 0\end{array}\right)$ for some $T_{0}, T_{1}$ in $M$. Since $[T P(q+\Sigma) H]=U[P(q+\Sigma) H]=[Q(q+\Sigma) H]$ and $\left[T^{-1} Q(q+\Sigma) H\right]=\left[A^{-1} U^{*} Q(q+\Sigma) H\right]=\left[A^{-1} P(q+\Sigma) H\right]=[P(q+\Sigma) H], \tilde{T} \in \operatorname{alg} \tilde{\mathcal{L}}$. Since $\tilde{T}^{2}=I$ and $\varepsilon$ is multiplicative on alg $\tilde{\mathcal{L}}$,

$$
\left(\begin{array}{cc}
0 & T_{1} \\
T_{0} & 0
\end{array}\right)^{2}=I
$$

hence $T_{0}$ is invertible in $M$. Let $T_{0}=W\left|T_{0}\right|$ be its polar decomposition. Then $W$ is a unitary operator in $M$. Since $\varepsilon(\tilde{T}) \in \tilde{\mathcal{L}}^{\prime}$ we get $T_{0} P(q+\Sigma)=Q(q+\Sigma) T_{0}$ for every $q \in \Gamma$. Hence

$$
\begin{aligned}
T_{0}^{*} T_{0} P(q+\Sigma) & =T_{0}^{*} Q(q+\Sigma) T_{0}=\left(Q(q+\Sigma) T_{0}\right)^{*} T_{0} \\
& =\left(T_{0} P(q+\Sigma)\right)^{*} T_{0}=P(q+\Sigma) T_{0}^{*} T_{0}
\end{aligned}
$$

so that $\left|T_{0}\right| \in \mathcal{L}^{\prime}$ and $W P(q+\Sigma) W^{*}=Q(q+\Sigma), q \in \Gamma$. Hence $B=W^{*} T$ satisfies $[B P(q+\Sigma) H]=W^{*}[T P(q+\Sigma) H]=[P(q+\Sigma) H]$ and, consequently, $B \in \operatorname{alg} \mathcal{L} \cap(\operatorname{alg} \mathcal{L})^{-1} \cap M$.

COROLlaRY 1. Let $\mathcal{L}$ be a commutative subspace lattice contained in a von Neumann algebra $M \subseteq B(H)$ and having a totally atomic core (that is, the von Neumann algebra generated by $\mathcal{L}$ is totally atomic). If $T \in M$ and $T=U A$ where $U \in B(H)$ is unitary and $A \in \operatorname{alg} \mathcal{L} \cap(\operatorname{alg} \mathcal{L})^{-1}$ then $U$ and $A$ can be chosen in $M$.

Proof. Using [4, Theorem 4.4] there is a compact abelian group $A$, a positive semigroup $\Sigma$ in $\Gamma=\hat{A}$ and a strongly continuous unitary representation $W=$ $\left\{W_{t}\right\}_{t \in A}$ on $H$ with $W_{t} \in M$ such that $\mathcal{L}$ is generated by $\{P(q+\Sigma): q \in \Gamma\}$, where $P(\cdot)$ is the projection valued measure associated with $\left\{W_{t}\right\}$. The corollary now follows immediately from the theorem.

Note that when $\mathcal{L}$ is a well-ordered nest this result was obtained in [9, Corollary 2.4].

With $\Gamma$ and $\Sigma$ as above $\Gamma$ is an ordered group where $p \leq q$ if and only if $q-p \in \Sigma$ and $p<q$ if $p \leq q, p \neq q$. If $\Sigma \cup(-\Sigma)=\Gamma$ then the order is linear. We say that the (linearly) ordered group $(\Gamma, \Sigma)$ is scattered if it does not contain any dense subset. (Here $\Lambda \subseteq \Gamma$ is dense in $\Gamma$ if for every $p<q, p, q$ in $\Gamma$, there is $\gamma \in \Lambda$ such that $p<\gamma<q$.$) If \Gamma=\mathbf{Z}^{n}$ and $\Sigma=\left\{\left(m_{1}, m_{2}, \ldots, m_{n}\right)=\bar{m}\right.$ : for some $k<n$, $m_{1}=\cdots=m_{k}=0$ and $m_{k+1}>0$ or $\left.\bar{m}=(0, \ldots, 0)\right\}$ then $(\Gamma, \Sigma)$ is scattered. In fact, every scattered countable ordered group is $\mathbf{Z}^{\gamma}$ where $\gamma$ is a countable ordinal (see [10, Theorem 8.5]). If $(\Gamma, \Sigma)$ is scattered then its completion (as a lattice) is countable (see [10, Exercise 5.33]).

Let $\alpha: A \rightarrow \operatorname{Aut}(M)$ be a $\sigma$-weakly continuous representation of $A$ as a group of *-automorphisms on a von Neumann algebra $M$. If $\Lambda$ is a (closed) subset of $\Gamma$ we write $M^{\alpha}(\Lambda)=\left\{T \in M: \operatorname{sp}_{\alpha}(T) \subseteq \Lambda\right\}$ where $\operatorname{sp}_{\alpha}(\cdot)$ is Arveson's spectrum. If $\Sigma \subseteq \Gamma$ is a positive semigroup then [7] $M(\Sigma)^{\alpha}$ is a $\sigma$-weakly closed algebra and we will refer to it as the analytic subalgebra of $M$ associated with $\alpha$ and $\Sigma$. We have $M^{\alpha}(\Sigma) \cap M^{\alpha}(\Sigma)^{*}=\left\{T \in M: \alpha_{t}(T)=T\right.$ for all $\left.t \in A\right\}$. 
In [11, Theorem 3.10] it is shown that when $A=\mathbf{T}$ (the unit circle) and $\Sigma=$ $\{n \in \mathbf{Z}: n \geq 0\}$ then every $T \in M$ can be factored with respect to $\left(M, M^{\alpha}(\Sigma)\right)$. The following corollary extends this result.

COROLlARY 2. Let $M$ be a $\sigma$-finite von Neumann algebra and $A$ be a compact abelian group whose dual, $\Gamma$, is a scattered linearly ordered group with a positive semigroup $\Sigma$. Let $\alpha$ be a $\sigma$-weakly continuous representation of $A$ as a group of *-automorphisms of $M$. Then every invertible operator $T$ in $M$ can be written as $U A$ where $U$ is a unitary operator in $M$ and $A \in M^{\alpha}(\Sigma) \cap M^{\alpha}(\Sigma)^{-1}$.

ProOF. Since $A$ is compact there is a faithful normal expectation $\varepsilon_{0}$ from $M$ onto the fixed point algebra $M^{\alpha}\left(=\left\{a \in M: \alpha_{t}(a)=a, t \in A\right\}\right)$; namely $\varepsilon_{0}(a)=\int_{A} \alpha_{t}(a) d t$. Therefore there exists a faithful normal $\left\{\alpha_{t}\right\}$-invariant state $\phi$ on $M$. Considering the Gelfand-Naimark-Segal construction of $\phi$ we may suppose that $M$ has a separating and cyclic vector $\xi_{0} \in H$ such that $\phi(x)=\left\langle x \xi_{0}, \xi_{0}\right\rangle$, $x \in M$. Since $\phi \circ \alpha_{t}=\phi, t \in A$, the map $a \xi_{0} \rightarrow \alpha_{t}(a) \xi_{0}, a \in M$, defines a unitary operator $W_{t}$ on $H$. Clearly $\left\{W_{t}\right\}$ is a strongly continuous unitary representation of $A$ on $H$ and $\alpha_{t}(a)=W_{t} a W_{t}^{*}$. By [4, Proposition 2.1], $M \cap$ alg $\mathcal{L}=M^{\alpha}(\Sigma)$ where $\mathcal{L}$ is the subspace lattice generated by $\{P(q+\Sigma): q \in \Gamma\}$ (and $P(\cdot)$ is the projection valued measure associated with $\left.\left\{W_{t}\right\}\right)$. Since $\Gamma$ is totally ordered, $\mathcal{L}$ is a nest and, since $\Gamma$ is scattered, $\mathcal{L}$ is countable (and complete).

Using [5, Proposition 4.1] we find that every invertible operator $T \in B(H)$ can be written as $U A$ where $U$ is a unitary operator and $A \in \operatorname{alg} \mathcal{L} \cap(\operatorname{alg} \mathcal{L})^{-1}$. Using the theorem we can choose $A, U$ in $M$, provided $T \in M$.

For the next corollary let $\alpha$ be an action of $A=\mathbf{T}^{n}$ on a $\sigma$-finite von Neumann algebra $M$. As in the proof of Corollary 2, we can represent $M$ on a Hilbert space $H$ such that there is a vector $\xi_{0}$ in $H$ that is a separating and cyclic vector for $M$, and for $a \in M, t \in A$, we have $\left\langle a \xi_{0}, \xi_{0}\right\rangle=\left\langle\alpha_{t}(a) \xi_{0}, \xi_{0}\right\rangle$.

COROLlARY 3. Let $\alpha, H$ and $M$ be as above and let $\Sigma=\left\{\left(m_{1}, m_{2}, \ldots, m_{n}\right) \in\right.$ $\left.\mathbf{Z}^{n}: m_{i} \geq 0\right\}$. Then, for an invertible operator $T \in M, T$ can be factored as $U A$ (where $U$ is a unitary operator and $A \in M^{\alpha}(\Sigma) \cap M^{\alpha}(\Sigma)^{-1}$ ) if and only if the projections onto the subspaces $\left\{\left[T M^{\alpha}(p+\Sigma) \xi_{0}\right]: p \in \Gamma\right\}$ pairwise commute.

ProOF. Note that $\left[M^{\alpha}(p+\Sigma) \xi_{0}\right]=[P(p+\Sigma) H]$ where $P(\cdot)$ is the projection valued measure of $\left\{W_{t}\right\}$ and $W_{t}$ is defined by $W_{t} a \xi_{0}=\alpha_{t}(a) \xi_{0}, a \in M$.

The proof now follows from [3, Theorem 4] and the theorem.

\section{REFERENCES}

1. W. B. Arveson, Analyticity in operator algebras, Amer. J. Math. 89 (1967), 578-642.

2. _ Interpolation problems in nest algebras, J. Funct. Anal. 20 (1975), 208-233.

3. J. Daughtry, Factorization along several nests, Preprint.

4. J. Kraus, $W^{*}$-dynamical systems and reflexive operator algebras, J. Operator Theory 8 (1982), 181-194.

5. D. R. Larson, Nest algebras and similarity transformations, Ann. of Math. (2) 121 (1985), 409-427.

6.

7. R. I. Loebl and P. S. Muhly, Analyticity and flows in von Neumann algebras, J. Funct. Anal. 29 (1978), 214-252. 
8. D. Pitts, Thesis, University of California at Berkeley, 1986.

9. S. Power, Factorization in analytic operator algebras, Preprint.

10. J. Rosenstein, Linear orderings, Academic Press, New York, 1982.

11. B. Solel, Analytic operator algebras (factorization and an expectation), Trans. Amer. Math. Soc. 287 (1985), 799-817.

Department of Mathematics, University of Pennsylvania, Philadelphia, PENNSYLVANIA 19104

Current address: Department of Mathematics, University of Haifa, Haifa, Israel 